\title{
Studies on physio-morphology, floral biology and fruit characteristics of mango
}

\author{
D. A. N. Majumder, L. Hassan ${ }^{1}$, M. A. Rahim ${ }^{2}$ and M. A. Kabir \\ Department of Biotechnology, ${ }^{1}$ Department of Genetics and Plant Breeding, ${ }^{2}$ Department of Horticulture, Bangladesh \\ Agricultural University, Mymensingh-2202, Bangladesh and ${ }^{3}$ Department of Horticulture, Hajee Md. Danesh Sci. \& \\ Tech. University, Dinajpur-5200, Bangladesh. Email: dilrumajumder@yahoo.com
}

\begin{abstract}
Plant, inflorescences and fruit characteristics of 60 mango genotypes were studied during the period 2007 to 2008. There were distinct variations among the findings of the gemplasm on plant, leaf, inflorescence fruit characters and yield. Wide variations were observed in relation to the $\%$ flowering shoot, $\%$ perfect flower, $\%$ fruit set per panicle, number of harvested fruits per plant, individual fruit weight, \% edible portion and \% total soluble solid ranging from 24.00 to $71.33 \%, 8.10$ to $19.17 \%, 9.07$ to $29.27 \%, 21.33$ to $60.33,365.33$ to $219.00 \mathrm{~g}, 45.22$ to $79.83 \%$ and 16.90 to $28.26 \%$, respectively. The germplasm MI28 was top of the list in case of number of panicle, number of main branch per panicle, percent perfect flower and fruit harvest per plant. The maximum and minimum number of fruit set per panicle was noted in MI28 and MI92, respectively. The maximum percentage of fruit harvest per panicle was found in MI94 (5.46) but the germplasm MI28 gave the highest number of fruit per plant (60.33). Moreover, the germplasm MI09 had the highest percentage of edible portion (79.83).
\end{abstract}

Keywords: Mangifera indica, Physio-morphology, Inflorescences, Fruit characteristics

\section{Introduction}

Mango (Mangifera indica L.), a member of the family Anacardiaceae, is one of the choicest fruits in the world (De Candolle, 1904 and Popenoe, 1927). It has medium calorific and high nutritional values. Mango grows in almost all parts of Bangladesh but the commercial and good quality grafted mangoes with known varietal identity are mostly confined in its North-Western districts. But the eastern area does not produce commercially any reputed variety. Moreover, studies relating to the performance of such varieties grown in those areas are scanty. The general impression is that elite mango varieties do not perform well when grown in the eastern areas. So, the need exists to assess the performance of elite varieties under that area. Again, characterization is an important aspect for documentation of the performance of the studied cultivars, which would help to introduce, select and improve the existing mango varieties. Therefore, an attempt was made to study the physio-morphology, inflorescences, fruit set and yields of 60 germplasm in the mango orchard of BAU- Germplasm Centre, Mymensingh.

\section{Materials and Methods}

The present experiment was conducted in a pre-established orchard named Germplasm (GP) Centre of Fruit Tree Improvement Project (FTIP, Department of Horticulture, Bangladesh Agricultural University, Mymensingh during October 2007 to September 2008. The experiment was conducted on 8-10 years old mango plants of 60 mango germplasm. The experiment was laid out in a RCBD with three replications, where a single uniform tree constituted the unit of replication. Both the distances between plant to plant and row to row were $6 \mathrm{~m}$. Irrigation was given after fruit set and thereafter at a fortnight interval. Fertilizers @ 21-15 kg FYM, $750 \mathrm{~g}$ urea, $400 \mathrm{~g}$ TSP, $250 \mathrm{~g} \mathrm{MP}, 250 \mathrm{~g}$ Gypsum and $15 \mathrm{~g}$ Zinc sulphate were applied per plant in two splits as per recommendation by Hossain (1989). Mature fruits were collected randomly from the selected plants. Ten fruits from each of the selected germplasm were taken in the laboratory for reading their physical characteristics like shape of fruit, external appearance, skin texture, fruit weight, length, breath, thickness, days to maturity, percent fruit harvest per panicle, number of fruit harvest per plant, weight of harvested fruit, edible portion, non-edible portion and stone weight. Data on different morphological parameters from 60 mango genotypes were recorded according to the descriptors for mango (IPGRI, 2003). The total soluble solids content of mango pulp was estimated by using Abbe Refreactometer. 


\section{Results and Discussion}

\section{Plant characteristics}

As evident from results (Table 1) a total of 23 genotypes showed ellipsoid plant shape and the rest of the genotypes were spheroid. The mango plants under the study showed two types of growth habit viz., spreading upright and intermediate. Most of the genotypes showed spreading and dense type growth habit and rest were upright and intermediate in nature. The mango plants under the study showed two types of bearing habit viz., alternate and regular. Only 9 genotypes showed regular type bearing habit in nature. In respect of plant height, significant variation was observed among genotypes. Hodgson (1967) found fruit trees were of three growth habit such as upright, spreading and open. The tallest plant (578.22 $\mathrm{cm})$ was observed in genotype MI58 followed by MI24 $(540.35 \mathrm{~cm})$ and MI29 $(540.29 \mathrm{~cm})$. The shortest plant $(211.31 \mathrm{~cm})$ was recorded in genotype MI98.

Table 1. Plant characteristics of 60 mango genotypes

\begin{tabular}{|c|c|c|c|c|c|}
\hline Genotypes & $\begin{array}{l}\text { Form/ Shape of } \\
\text { plant }\end{array}$ & Growth habit of plant & $\begin{array}{l}\text { Density of } \\
\text { branches }\end{array}$ & $\begin{array}{l}\text { Bearing } \\
\text { habit }\end{array}$ & $\begin{array}{l}\text { Plant height } \\
\text { (cm.) }\end{array}$ \\
\hline $\mathrm{MI01}$ & Ellipsoid & Intermediate & Sparse & Regular & 313.80 \\
\hline $\mathrm{MIO2}$ & Ellipsoid & Intermediate & Sparse & Alternate & 345.98 \\
\hline $\mathrm{MIO3}$ & Spheroid & Spreading & Dense & Alternate & 229.09 \\
\hline $\mathrm{MIO4}$ & Ellipsoid & Intermediate & Sparse & Regular & 295.69 \\
\hline MI08 & Ellipsoid & Spreading & Dense & Alternate & 291.87 \\
\hline MI09 & Ellipsoid & Intermediate & Sparse & Alternate & 355.37 \\
\hline MI12 & Spheroid & Spreading & Dense & Alternate & 303.02 \\
\hline MI16 & Ellipsoid & Upright & Sparse & Alternate & 370.03 \\
\hline MI19 & Ellipsoid & Intermediate & Dense & Alternate & 365.58 \\
\hline MI20 & Ellipsoid & Intermediate & Dense & Regular & 373.76 \\
\hline MI21 & Ellipsoid & Intermediate & Sparse & Alternate & 377.33 \\
\hline MI22 & Spheroid & Spreading & Dense & Alternate & 386.82 \\
\hline MI23 & Spheroid & Spreading & Dense & Alternate & 391.47 \\
\hline MI24 & Spheroid & Spreading & Dense & Alternate & 540.35 \\
\hline MI25 & Spheroid & Spreading & Sparse & Alternate & 524.45 \\
\hline MI26 & Ellipsoid & Intermediate & Dense & Alternate & 441.85 \\
\hline MI27 & Spheroid & Spreading & Dense & Alternate & 517.13 \\
\hline MI28 & Spheroid & Intermediate & Dense & Regular & 295.70 \\
\hline MI29 & Ellipsoid & Upright & Sparse & Alternate & 540.29 \\
\hline MI33 & Ellipsoid & Upright & Sparse & Alternate & 444.64 \\
\hline MI38 & Spheroid & Spreading & Dense & Alternate & 361.20 \\
\hline MI39 & Spheroid & Spreading & Dense & Alternate & 270.44 \\
\hline $\mathrm{MI} 40$ & Spheroid & Spreading & Dense & Alternate & 246.32 \\
\hline MI41 & Spheroid & Spreading & Dense & Alternate & 285.89 \\
\hline $\mathrm{MI} 43$ & Spheroid & Spreading & Dense & Regular & 314.05 \\
\hline $\mathrm{Ml} 44$ & Ellipsoid & Intermediate & Sparse & Alternate & 255.71 \\
\hline MI45 & Ellipsoid & Intermediate & Sparse & Alternate & 285.15 \\
\hline MI46 & \begin{tabular}{|l|} 
Ellipsoid \\
\end{tabular} & Intermediate & Sparse & Alternate & 312.63 \\
\hline $\mathrm{Ml} 47$ & \begin{tabular}{|l|} 
Ellipsoid \\
\end{tabular} & Intermediate & Sparse & Alternate & 410.39 \\
\hline MI48 & Ellipsoid & Intermediate & Sparse & Alternate & 440.84 \\
\hline MI49 & Spheroid & Spreading & Dense & Regular & 246.20 \\
\hline MI50 & Spheroid & Upright & Sparse & Alternate & 339.04 \\
\hline MI51 & Spheroid & Spreading & Dense & Alternate & 355.22 \\
\hline MI52 & Spheroid & Spreading & Dense & Alternate & 277.96 \\
\hline MI54 & Ellipsoid & Intermediate & Dense & Alternate & 422.77 \\
\hline MI58 & Ellipsoid & Upright & Sparse & Alternate & 578.22 \\
\hline MI60 & Ellipsoid & Upright & Sparse & Alternate & 483.96 \\
\hline MI61 & Spheroid & Spreading & Sparse & Alternate & 390.51 \\
\hline MI64 & Spheroid & Spreading & Dense & Alternate & 250.54 \\
\hline
\end{tabular}


Table 1 Contd.

\begin{tabular}{|c|c|c|c|c|c|}
\hline Genotypes & $\begin{array}{c}\text { Form/ Shape of } \\
\text { plant }\end{array}$ & Growth habit of plant & $\begin{array}{c}\text { Density of } \\
\text { branches }\end{array}$ & Bearing habit & $\begin{array}{l}\text { Plant height } \\
(\mathrm{cm} .)\end{array}$ \\
\hline MI70 & Spheroid & Spreading & Dense & Alternate & 328.59 \\
\hline MI74 & Spheroid & Spreading & Sparse & Alternate & 375.31 \\
\hline MI75 & Spheroid & Spreading & Dense & Alternate & 287.25 \\
\hline MI77 & Spheroid & Spreading & Sparse & Alternate & 371.08 \\
\hline MI80 & Ellipsoid & Upright & Sparse & Alternate & 409.11 \\
\hline MI81 & Spheroid & Spreading & Dense & Alternate & 403.26 \\
\hline MI82 & Spheroid & Spreading & Dense & Alternate & 228.28 \\
\hline MI83 & Spheroid & Spreading & Sparse & Alternate & 258.46 \\
\hline MI84 & Spheroid & Spreading & Dense & Alternate & 328.83 \\
\hline MI85 & Spheroid & Spreading & Sparse & Alternate & 226.05 \\
\hline MI86 & Spheroid & Spreading & Dense & Alternate & 308.41 \\
\hline MI88 & Spheroid & Spreading & Dense & Regular & 365.16 \\
\hline MI90 & Spheroid & Spreading & Dense & Alternate & 322.79 \\
\hline MI91 & Ellipsoid & Upright & Sparse & Alternate & 389.99 \\
\hline MI92 & Ellipsoid & Upright & Sparse & Alternate & 399.18 \\
\hline MI93 & Ellipsoid & Upright & Sparse & Alternate & 291.23 \\
\hline MI94 & Spheroid & Spreading & Dense & Regular & 410.76 \\
\hline MI95 & Spheroid & Spreading & Dense & Regular & 447.08 \\
\hline MI96 & Spheroid & Spreading & Sparse & Alternate & 397.24 \\
\hline MI97 & Spheroid & Spreading & Dense & Alternate & 408.53 \\
\hline MI98 & Spheroid & Spreading & Dense & Alternate & 211.31 \\
\hline Range & - & - & - & - & $211.31-578.22$ \\
\hline Mean & - & - & - & - & 356.66 \\
\hline CV\% & - & - & - & - & $9.36 \%$ \\
\hline SE & - & - & - & - & 19.27 \\
\hline $\operatorname{LSD}(0.01 \%)$ & - & - & - & - & 71.34 \\
\hline
\end{tabular}

\section{Leaf characteristics}

The result showed significant variation in leaf characters of mango genotypes (Table 2). Leaf length, leaf width and petiole length varied from 113.16 to $35.82 \mathrm{~cm}, 3.59$ to $9.76 \mathrm{~cm}$ and 2.53 to $5.33 \mathrm{~cm}$, respectively. Leaf area varied significantly among the genotypes. The genotype MI24 had the highest leaf area $\left(74.32 \mathrm{~cm}^{2}\right)$ which was statistically different from rest of the genotypes. The lowest leaf area was recorded in genotype MI98 (Table 2).

\section{Inflorescence characteristics}

The inflorescence colour in most of the varieties varied from light green to light green with red patch (Table $3 a$ ) and the position varied from terminal axillaries to terminal. Duration of flowering ranged from 17.67 to 35.33 days. Majumder and Sharma (1990) reported that the flowering time varied with the varieties and growing locations. The maximum percentage of flowering shoot was recorded in the accession MI28 (71.33 \%) and the maximum panicle (2.77) was also found in the accession MI28. Islam et al. (1995) also observed variation in number of panicles per shoot among different mango varieties. The number of main branches per panicle ranged from 21.57 to 52.53 and the germplasm MI94 had the highest number (52.53) of main branches per panicle (Table 3b). The percent of perfect flower ranged from 8.10 to $19.77 \%$. Mukherjee (1997) reported that the ratio of male to perfect flower was strongly influenced by environmental and cultural factors. The highest percentage of fruit set (29.27\%) was recorded in MI28. The maximum dropping (88.43\%) was recorded in MI39 (Table 3b). 
Table 2. Leaf characteristics of 60 mango genotypes

\begin{tabular}{|c|c|c|c|c|c|c|}
\hline Genotypes & $\begin{array}{l}\text { Length of } \\
\text { lamina } \\
(\mathrm{cm})\end{array}$ & $\begin{array}{c}\text { Width of } \\
\text { lamina } \\
(\mathrm{cm})\end{array}$ & $\begin{array}{l}\text { Length of } \\
\text { petiole } \\
(\mathrm{cm})\end{array}$ & $\begin{array}{l}\text { Area of leaf } \\
\left(\mathrm{cm}^{2}\right)\end{array}$ & Leaf shape & $\begin{array}{l}\text { Leaf } \\
\text { margin }\end{array}$ \\
\hline MI01 & 18.28 & 5.56 & 4.22 & 68.52 & Lanceolate & Flat \\
\hline $\mathrm{MIO2}$ & 16.70 & 3.59 & 2.65 & 58.31 & Lanceolate & wavy \\
\hline $\mathrm{MIO3}$ & 21.21 & 4.85 & 5.33 & 69.83 & Elliptic-anceolate & Wavy \\
\hline $\mathrm{MIO4}$ & 16.93 & 4.87 & 2.84 & 66.62 & Oblong-anceolate & Flat \\
\hline MI08 & 15.68 & 4.02 & 2.74 & 61.05 & Lanceolate & Wavy \\
\hline MI09 & 16.73 & 4.81 & 3.22 & 64.53 & Lanceolate & Flat \\
\hline MI12 & 16.01 & 4.32 & 2.87 & 59.31 & Lanceolate & Flat \\
\hline MI16 & 23.81 & 5.08 & 3.98 & 64.77 & Lanceolate & Flat \\
\hline MI19 & 18.38 & 5.01 & 5.21 & 57.38 & Elliptic-Laceolate & Wavy \\
\hline MI20 & 22.65 & 6.49 & 4.72 & 64.78 & Oblong-anceolate & Crinkled \\
\hline MI21 & 18.40 & 5.15 & 4.05 & 60.11 & Elliptic-lanceolate & Wavy \\
\hline MI22 & 17.49 & 4.87 & 4.21 & 67.79 & Elliptic Lanceola & Flat \\
\hline MI23 & 21.46 & 3.97 & 4.66 & 60.99 & Ellip-Lance. & Wavy \\
\hline MI24 & 35.82 & 9.76 & 4.88 & 74.32 & Oblo-Lance. & Wavy \\
\hline MI25 & 27.47 & 6.21 & 3.30 & 65.29 & Lanceolate & Flat \\
\hline MI26 & 21.59 & 5.14 & 4.15 & 61.02 & Ellip-Lance. & Wavy \\
\hline MI27 & 31.77 & 5.99 & 4.35 & 71.39 & Lanceolate & Flat \\
\hline MI28 & 15.44 & 5.17 & 3.70 & 54.19 & Lanceolate & Wavy \\
\hline MI29 & 17.50 & 4.95 & 2.66 & 59.62 & Lanceolate & Wavy \\
\hline MI33 & 13.16 & 4.84 & 2.66 & 55.75 & Lanceolate & Flat \\
\hline MI38 & 14.83 & 4.14 & 2.96 & 54.91 & Lanceolate & Flat \\
\hline MI39 & 14.92 & 4.67 & 3.84 & 55.96 & Lanceolate & Flat \\
\hline $\mathrm{MI40}$ & 18.87 & 4.57 & 3.66 & 56.42 & Lanceolate & Wavy \\
\hline MI41 & 13.77 & 5.00 & 2.74 & 53.65 & Ellip-Lance. & Flat \\
\hline MI43 & 18.56 & 4.67 & 3.53 & 62.39 & Lanceolate & Flat \\
\hline MI44 & 17.21 & 4.85 & 3.82 & 58.80 & Oblo-Lance. & Flat \\
\hline MI45 & 13.78 & 4.98 & 2.77 & 53.55 & Oblo-Lance. & Flat \\
\hline MI46 & 15.99 & 4.62 & 2.77 & 56.52 & Lanceolate & Flat \\
\hline $\mathrm{MI} 47$ & 14.62 & 5.52 & 2.99 & 56.39 & Oblo-Lance. & Flat \\
\hline MI48 & 16.82 & 4.49 & 2.92 & 55.29 & Lanceolate & Flat \\
\hline MI49 & 19.50 & 4.34 & 2.86 & 57.65 & Lanceolate & Flat \\
\hline MI50 & 15.43 & 3.89 & 3.52 & 52.82 & Lanceolate & Flat \\
\hline MI51 & 16.87 & 3.86 & 3.59 & 53.95 & Lanceolate & Flat \\
\hline MI52 & 17.33 & 4.51 & 3.54 & 55.07 & Ellip-Lance. & Wavy \\
\hline MI54 & 18.48 & 5.86 & 3.77 & 57.12 & Ellip-Lance. & Flat \\
\hline MI58 & 32.65 & 6.21 & 4.55 & 71.89 & Lanceolate & Flat \\
\hline MI60 & 19.63 & 5.84 & 4.59 & 62.41 & Elliptic & Wavy \\
\hline MI61 & 30.38 & 6.25 & 4.70 & 71.30 & Ellip-Lance. & Wavy \\
\hline MI64 & 15.85 & 4.82 & 3.72 & 54.70 & Ellip-Lance. & Wavy \\
\hline MI70 & 21.59 & 5.69 & 3.59 & 61.65 & Lanceolate & Flat \\
\hline MI74 & 16.24 & 5.75 & 3.84 & 58.67 & Lanceolate & Wavy \\
\hline MI75 & 19.88 & 5.12 & 3.51 & 61.65 & Lanceolate & Wavy \\
\hline MI77 & 22.39 & 5.75 & 3.99 & 63.27 & Lanceolate & Flat \\
\hline MI80 & 17.78 & 5.75 & 3.73 & 62.55 & Lanceolate & Flat \\
\hline MI81 & 19.49 & 5.43 & 3.11 & 61.71 & Ellip-Lance. & Flat \\
\hline MI82 & 23.50 & 5.64 & 4.61 & 63.02 & Lanceolate & Flat \\
\hline MI83 & 17.03 & 5.07 & 4.01 & 58.44 & Ellip-Lance. & Flat \\
\hline MI84 & 19.08 & 5.72 & 3.89 & 55.00 & Lanceolate & Wavy \\
\hline MI85 & 16.73 & 4.90 & 4.05 & 59.45 & Ellip-Lance. & Flat \\
\hline MI86 & 17.84 & 4.89 & 4.17 & 59.08 & Lanceolate & Flat \\
\hline MI88 & 13.98 & 5.03 & 3.56 & 53.09 & Lanceolate & Flat \\
\hline MI90 & 28.39 & 6.55 & 3.69 & 66.06 & Lanceolate & Flat \\
\hline MI91 & 18.84 & 6.10 & 3.85 & 61.68 & Ellip-Lance & Flat \\
\hline
\end{tabular}


Table 2 Contd.

\begin{tabular}{|l|c|c|c|c|l|l|}
\hline Genotypes & $\begin{array}{c}\text { Length of } \\
\text { lamina } \\
(\mathrm{cm})\end{array}$ & $\begin{array}{c}\text { Width of } \\
\text { lamina } \\
(\mathrm{cm})\end{array}$ & $\begin{array}{c}\text { Length of } \\
\text { petiole } \\
(\mathrm{cm})\end{array}$ & $\begin{array}{c}\text { Area of leaf } \\
\left(\mathrm{cm}^{2}\right)\end{array}$ & Leaf shape & Leaf margin \\
\hline MI92 & 18.40 & 4.87 & 4.10 & 55.41 & Lanceolate & Flat \\
\hline MI93 & 23.48 & 5.91 & 3.60 & 62.38 & Lanceolate & Flat \\
\hline MI94 & 17.04 & 5.66 & 3.77 & 54.84 & Lanceolate & Flat \\
\hline MI95 & 25.19 & 6.82 & 4.15 & 65.44 & Lanceolate & Flat \\
\hline MI96 & 18.13 & 5.60 & 3.48 & 61.60 & Lanceolate & Flat \\
\hline M197 & 19.73 & 3.94 & 2.53 & 55.15 & Lanceolate & Flat \\
\hline M198 & 15.86 & 3.79 & 2.75 & 52.59 & Lanceolate & Wavy \\
\hline Range & $13.16-35.82$ & $3.59-9.76$ & $2.53-5.33$ & $52.59-74.32$ & - & - \\
\hline Mean & 19.38 & 5.17 & 3.69 & 60.30 & - & - \\
\hline CV (\%) & 7.83 & 7.35 & 6.48 & 5.71 & - & - \\
\hline SE & 0.88 & 0.22 & 0.14 & 1.99 & - & - \\
\hline LSD $(0.01 \%)$ & 3.24 & 0.812 & 0.51 & 7.37 & - & - \\
\hline
\end{tabular}

Table 3a. Inflorescence characteristics of 60 mango genotypes

\begin{tabular}{|c|c|c|c|c|c|}
\hline Genotypes & $\begin{array}{l}\text { Position of } \\
\text { inflorescence }\end{array}$ & $\begin{array}{l}\text { Colour of } \\
\text { inflorescence }\end{array}$ & Type of flower & $\begin{array}{l}\text { Duration of } \\
\text { flowering } \\
\text { (days) }\end{array}$ & $\begin{array}{c}\text { Flowering } \\
\text { shoot } \\
(\%)\end{array}$ \\
\hline MI01 & Terminal & Light green & Tetramerous & 34.00 & 67.33 \\
\hline $\mathrm{MIO2}$ & Terminal & Light green & Tetramerous & 29.33 & 67.33 \\
\hline MI03 & Terminal \& Axillaries & Light green & Pentamerous & 22.00 & 61.67 \\
\hline $\mathrm{MI04}$ & Terminal \& Axillaries & Green with red patches & Tetra \&Pentamerous & 21.00 & 53.67 \\
\hline MI08 & Terminal \& Axillaries & Green with red patches & Pentamerous & 18.67 & 46.33 \\
\hline MI09 & Terminal & Light green & Pentamerous & 18.33 & 48.67 \\
\hline MI12 & Terminal \& Axillaries & Green with red patches & Pentamerous & 24.33 & 57.33 \\
\hline MI16 & Terminal & Green with red patches & Pentamerous & 33.33 & 31.33 \\
\hline MI19 & Terminal \& Axillaries & Green with red patches & Pentamerous & 19.00 & 51.33 \\
\hline $\mathrm{MI} 20$ & Terminal \& Axillaries & Green with red patches & Pentamerous & 31.00 & 43.00 \\
\hline MI21 & Terminal \& Axillaries & Light green & Pentamerous & 20.33 & 39.67 \\
\hline MI22 & Terminal \& Axillaries & Green with red patches & Pentamerous & 25.67 & 32.00 \\
\hline MI23 & Terminal & Green with red patches & Pentamerous & 20.67 & 32.67 \\
\hline MI24 & Terminal \& Axillaries & Green with red patches & Tetra\& Pentamerous & 20.67 & 41.33 \\
\hline MI25 & Terminal \& Axillaries & Green with red patches & Pentamerous & 34.67 & 44.67 \\
\hline MI26 & Terminal & Green with red patches & Pentamerous & 20.00 & 32.00 \\
\hline MI27 & Terminal \& Axillaries & Green with red patches & Pentamerous & 35.33 & 52.33 \\
\hline MI28 & Terminal \& Axillaries & Green with red patches & Pentamerous & 29.67 & 71.33 \\
\hline MI29 & Terminal & Light green & Tetra \& Pentamerous & 19.67 & 33.67 \\
\hline MI33 & Terminal & Dark red & Tetra\& Pentamerous & 19.67 & 61.00 \\
\hline MI38 & Terminal \& Axillaries & Light green & Pentamerous & 22.00 & 38.00 \\
\hline MI39 & Terminal & Light brick red & Pentamerous & 18.67 & 32.33 \\
\hline MI40 & Terminal \& Axillaries & Light green & Tetra\& Pentamerous & 25.33 & 31.00 \\
\hline MI41 & Terminal \& Axillaries & Light green & Tetra\& Pentamerous & 23.33 & 34.00 \\
\hline MI43 & Terminal & Green with red patches & Tetra\& Pentamerous & 18.67 & 31.00 \\
\hline MI44 & Terminal & Green with red patches & Pentamerous & 18.33 & 33.67 \\
\hline MI45 & Terminal & Green with red patches & Pentamerous & 31.33 & 39.67 \\
\hline MI46 & Terminal & Light green & Pentamerous & 17.67 & 56.67 \\
\hline MI47 & Terminal & Light green & Pentamerous & 29.33 & 36.00 \\
\hline MI48 & Terminal & Green with red patches & Tetra \& Pentamerous & 22.67 & 33.33 \\
\hline MI49 & Terminal \& Axillaries & Green with red patches & Pentamerous & 23.67 & 36.33 \\
\hline MI50 & Terminal & Light red & \begin{tabular}{|l|} 
Pentamerous \\
\end{tabular} & 30.00 & 32.33 \\
\hline MI51 & Terminal \& Axillaries & Green with red patches & Pentamerous & 22.67 & 33.67 \\
\hline MI52 & Terminal \& Axillaries & Light green & Pentamerous & 22.00 & 32.67 \\
\hline MI54 & Terminal \& Axillaries & Light green & Pentamerous & 22.00 & 32.67 \\
\hline
\end{tabular}


Table 3a. Contd.

\begin{tabular}{|c|c|c|c|c|c|}
\hline Genotypes & $\begin{array}{l}\text { Position of } \\
\text { inflorescence }\end{array}$ & $\begin{array}{l}\text { Colour of } \\
\text { inflorescence }\end{array}$ & Type of flower & $\begin{array}{c}\text { Duration of } \\
\text { flowering } \\
\text { (days) }\end{array}$ & $\begin{array}{c}\text { Flowering } \\
\text { shoot } \\
(\%)\end{array}$ \\
\hline MI58 & Terminal \& Axillaries & Green with red patches & Tetra\& Pentamerous & 24.33 & 34.00 \\
\hline MI60 & Terminal & Light green & Pentamerous & 23.00 & 43.00 \\
\hline MI61 & Terminal & Light green & Tetra\& Pentamerous & 17.67 & 35.33 \\
\hline MI64 & Terminal \& Axillaries & Green with red patches & Tetra\& Pentamerous & 31.00 & 47.67 \\
\hline MI70 & Terminal \& Axillaries & Light red & Pentamerous & 26.00 & 36.67 \\
\hline MI74 & Terminal \& Axillaries & Green with red patches & Pentamerous & 27.33 & 46.67 \\
\hline MI75 & Terminal \& Axillaries & Green with red patches & Tetra\& Pentamerous & 26.33 & 47.00 \\
\hline MI77 & Terminal & Green with red patches & Pentamerous & 30.00 & 31.67 \\
\hline MI80 & Terminal \& Axillaries & Green with red patches & Tetra\& Pentamerous & 21.33 & 24.00 \\
\hline MI81 & Terminal & Light green & Pentamerous & 30.33 & 64.33 \\
\hline MI82 & Terminal & Green with red patches & Pentamerous & 24.33 & 59.33 \\
\hline MI83 & Terminal \& Axillaries & Dark red & Pentamerous & 23.67 & 31.33 \\
\hline MI84 & Terminal & Green with red patches & Tetra\&Pentamerous & 24.67 & 29.67 \\
\hline MI85 & Terminal \& Axillaries & Light green & Pentamerous & 25.67 & 40.33 \\
\hline MI86 & Terminal \& Axillaries & Dark red & Pentamerous & 21.67 & 30.00 \\
\hline MI88 & Terminal \& Axillaries & Green with red patches & Tetra \& Pentamerous & 22.00 & 58.33 \\
\hline MI90 & Terminal & Light green & Pentamerous & 27.33 & 35.33 \\
\hline MI91 & Terminal \& Axillaries & Green with red patches & Pentamerous & 30.67 & 36.67 \\
\hline MI92 & Terminal \& Axillaries & Green with red patches & Pentamerous & 29.00 & 60.67 \\
\hline MI93 & Terminal & Green with red patches & Pentamerous & 21.00 & 33.67 \\
\hline MI94 & Terminal & Green with red patches & Pentamerous & 20.67 & 34.33 \\
\hline MI95 & Terminal \& Axillaries & Green with red patches & Pentamerous & 21.67 & 32.00 \\
\hline MI96 & Terminal \& Axillaries & Green with red patches & Pentamerous & 33.33 & 33.33 \\
\hline MI97 & Terminal \& Axillaries & Green with red patches & Pentamerous & 30.00 & 36.67 \\
\hline MI98 & Terminal \& Axillaries & Green with red patches & Pentamerous & 23.33 & 62.33 \\
\hline Range & - & - & - & $17.67-35.33$ & $24.00-71.33$ \\
\hline Mean & - & - & - & 24.68 & 42.41 \\
\hline CV (\%) & - & - & - & 10.98 & 7.97 \\
\hline SE & - & - & - & 1.57 & 1.95 \\
\hline $\begin{array}{l}\operatorname{LSD}(0.01 \\
\%)\end{array}$ & - & - & - & 5.79 & 7.22 \\
\hline
\end{tabular}

Table 3b. Panicle characteristics of 60 mango genotypes

\begin{tabular}{|l|c|c|c|c|c|c|}
\hline Genotypes & $\begin{array}{c}\text { Number of } \\
\text { Panicle per } \\
\text { shoot } \\
(\mathrm{no})\end{array}$ & $\begin{array}{c}\text { Length of } \\
\text { the panicle } \\
(\mathrm{cm})\end{array}$ & $\begin{array}{c}\text { Number of main } \\
\text { branch per } \\
\text { panicle (no) }\end{array}$ & $\begin{array}{c}\text { Percent } \\
\text { perfect } \\
\text { flower }\end{array}$ & $\begin{array}{c}\text { Percent fruit } \\
\text { set per panicle }\end{array}$ & $\begin{array}{c}\text { Dropping at } \\
\text { Initial stage } \\
(\%)\end{array}$ \\
\hline MI01 & 2.67 & 42.17 & 49.93 & 17.27 & 24.93 & 74.31 \\
\hline MI02 & 2.63 & 22.17 & 33.67 & 11.17 & 26.37 & 72.85 \\
\hline MI03 & 1.83 & 31.05 & 25.37 & 9.67 & 18.60 & 76.75 \\
\hline MI04 & 1.67 & 39.79 & 30.03 & 10.13 & 11.00 & 77.42 \\
\hline MI08 & 1.60 & 31.08 & 31.57 & 15.23 & 24.74 & 81.19 \\
\hline MI09 & 1.37 & 26.76 & 29.47 & 17.30 & 13.97 & 79.09 \\
\hline MI12 & 1.63 & 29.48 & 50.20 & 15.47 & 20.40 & 87.26 \\
\hline MI16 & 1.53 & 33.26 & 32.50 & 12.90 & 23.40 & 86.41 \\
\hline MI19 & 1.50 & 33.06 & 21.93 & 13.33 & 10.43 & 80.65 \\
\hline MI20 & 1.23 & 27.61 & 24.77 & 14.30 & 9.73 & 77.88 \\
\hline MI21 & 1.37 & 24.34 & 22.73 & 14.73 & 19.23 & 87.48 \\
\hline MI22 & 1.47 & 26.14 & 28.20 & 8.53 & 17.67 & 79.44 \\
\hline MI23 & 1.30 & 32.52 & 26.63 & 11.70 & 26.33 & 73.91 \\
\hline MI24 & 2.70 & 28.35 & 22.63 & 16.33 & 20.17 & 78.90 \\
\hline MI25 & 2.20 & 30.30 & 24.30 & 9.27 & 18.63 & 74.75 \\
\hline
\end{tabular}


Table 3b. Contd.

\begin{tabular}{|c|c|c|c|c|c|c|}
\hline Genotypes & $\begin{array}{c}\text { Number of } \\
\text { Panicle per } \\
\text { shoot } \\
\text { (no) }\end{array}$ & $\begin{array}{l}\text { Length of } \\
\text { the panicle } \\
(\mathrm{cm})\end{array}$ & $\begin{array}{l}\text { Number of main } \\
\text { branch per } \\
\text { panicle (no) }\end{array}$ & $\begin{array}{l}\text { Percent } \\
\text { perfect } \\
\text { flower }\end{array}$ & $\begin{array}{l}\text { Percent fruit } \\
\text { set per panicle }\end{array}$ & $\begin{array}{c}\text { Dropping at } \\
\text { Initial stage } \\
(\%)\end{array}$ \\
\hline MI26 & 1.40 & 31.32 & 25.40 & 12.60 & 13.83 & 72.27 \\
\hline MI27 & 1.23 & 28.57 & 23.83 & 9.93 & 28.80 & 75.05 \\
\hline MI28 & 2.77 & 26.27 & 52.20 & 19.17 & 29.27 & 69.90 \\
\hline MI29 & 1.90 & 32.50 & 27.20 & 15.00 & 11.47 & 80.22 \\
\hline MI33 & 2.47 & 27.98 & 23.50 & 12.27 & 27.00 & 82.40 \\
\hline MI38 & 1.23 & 25.85 & 31.57 & 8.40 & 22.07 & 81.79 \\
\hline MI39 & 1.57 & 33.20 & 38.37 & 9.37 & 10.40 & 88.43 \\
\hline MI40 & 1.53 & 27.87 & 22.31 & 12.10 & 16.70 & 86.59 \\
\hline MI41 & 1.23 & 26.08 & 44.47 & 14.33 & 16.50 & 71.89 \\
\hline MI43 & 1.63 & 32.24 & 24.53 & 12.33 & 22.30 & 84.53 \\
\hline MI44 & 2.23 & 32.34 & 21.93 & 10.17 & 11.83 & 73.97 \\
\hline MI45 & 2.37 & 38.75 & 50.97 & 11.10 & 20.67 & 70.13 \\
\hline MI46 & 2.30 & 24.79 & 25.60 & 12.67 & 24.60 & 69.20 \\
\hline MI47 & 2.07 & 17.75 & 25.60 & 13.23 & 14.03 & 84.92 \\
\hline MI48 & 2.47 & 27.64 & 22.27 & 8.10 & 19.37 & 83.20 \\
\hline MI49 & 2.50 & 36.57 & 49.87 & 9.10 & 20.43 & 86.55 \\
\hline MI50 & 2.07 & 23.25 & 22.77 & 9.57 & 11.47 & 82.66 \\
\hline MI51 & 1.40 & 32.54 & 21.57 & 9.37 & 15.40 & 77.95 \\
\hline MI52 & 1.63 & 37.41 & 26.33 & 13.43 & 17.03 & 71.13 \\
\hline MI54 & 1.23 & 37.46 & 22.70 & 14.97 & 27.50 & 72.87 \\
\hline MI58 & 1.37 & 38.80 & 21.93 & 9.87 & 11.77 & 82.59 \\
\hline MI60 & 1.23 & 24.35 & 25.23 & 10.57 & 15.73 & 82.57 \\
\hline MI61 & 1.60 & 38.42 & 33.13 & 16.20 & 25.73 & 69.42 \\
\hline MI64 & 2.07 & 29.71 & 36.47 & 12.20 & 21.23 & 75.02 \\
\hline MI70 & 1.50 & 30.71 & 30.90 & 12.07 & 27.80 & 70.61 \\
\hline MI74 & 1.70 & 37.97 & 29.00 & 13.20 & 19.33 & 74.61 \\
\hline Ml75 & 1.80 & 27.04 & 30.87 & 9.80 & 10.87 & 87.80 \\
\hline MI77 & 1.60 & 34.00 & 21.93 & 13.23 & 15.20 & 85.94 \\
\hline MI80 & 2.10 & 27.65 & 27.67 & 15.17 & 18.53 & 81.04 \\
\hline MI81 & 2.27 & 30.21 & 49.37 & 9.93 & 25.10 & 87.41 \\
\hline MI82 & 1.37 & 38.57 & 43.57 & 8.23 & 19.60 & 85.16 \\
\hline MI83 & 1.87 & 29.96 & 32.97 & 9.53 & 17.20 & 84.46 \\
\hline MI84 & 2.50 & 29.83 & 29.63 & 10.10 & 10.87 & 85.36 \\
\hline MI85 & 1.37 & 37.91 & 37.13 & 10.73 & 16.60 & 78.32 \\
\hline MI86 & 1.23 & 34.13 & 26.57 & 8.77 & 26.23 & 80.87 \\
\hline MI88 & 1.50 & 34.42 & 30.67 & 9.90 & 11.00 & 83.79 \\
\hline MI90 & 1.23 & 24.47 & 25.00 & 8.73 & 18.23 & 87.74 \\
\hline MI91 & 2.40 & 31.28 & 27.17 & 14.40 & 26.73 & 70.51 \\
\hline MI92 & 1.13 & 35.14 & 31.07 & 12.07 & 9.07 & 81.61 \\
\hline MI93 & 1.63 & 27.66 & 22.13 & 9.50 & 20.17 & 71.16 \\
\hline MI94 & 1.67 & 26.57 & 52.53 & 18.43 & 26.33 & 75.88 \\
\hline MI95 & 1.30 & 29.12 & 51.73 & 16.33 & 10.23 & 74.38 \\
\hline MI96 & 1.37 & 24.18 & 24.50 & 9.60 & 10.20 & 79.52 \\
\hline MI97 & 1.23 & 38.52 & 27.77 & 9.63 & 11.20 & 70.72 \\
\hline MI98 & 1.57 & 28.79 & 22.63 & 10.90 & 9.47 & 77.15 \\
\hline Range & $1.13-2.77$ & $17.75-42.17$ & $21.57-52.53$ & $8.10-19.17$ & $9.07-29.27$ & $69.90-88.43$ \\
\hline Mean & 1.74 & 30.79 & 30.78 & 12.06 & 18.50 & 78.96 \\
\hline $\mathrm{CV}(\%)$ & 7.89 & 11.17 & 4.08 & 12.44 & 7.35 & 4.80 \\
\hline SE & 0.08 & 1.99 & 0.73 & 0.87 & 0.79 & 2.19 \\
\hline LSD (0.01\%) & 0.29 & 7.35 & 2.69 & 3.21 & 2.90 & 8.10 \\
\hline
\end{tabular}




\section{Fruit characteristics}

Maximum number of days (178.67) was required for fruit maturity of the germplasm MI28, which was significantly different from those of the other germplasm (Table 4a). These results were found similar to the observation of Hossain (1989) who reported that mango under Bangladesh conditions takes about four to six month to reach maturity after flowering. These findings differed from that of Sardar et al. (1998) who consented that harvesting time varied from 92 to 134 days in some popular mango cultivars under the climatic conditions of Rajshahi. This might be due to environmental fluctuation over the year and the locality. Mukherjee (1997) observed the yellowish green to bright yellow skin colour of the fruits of ripened mango and also reported that fruit colour at maturity was dependent on genotype. The highest percentage of fruit harvested per panicle was in MI94 (5.46 \%) but the highest number of fruits was observed in MI28 (60.33). The highest weight of harvested fruits was found in MI94 (25.04), which was closely preceded by MI28 $(23.12 \mathrm{~kg})$. The shape of fruit of mango genotypes was classified into four groups' viz., Elliptic, Oblong, Round and Roundish. The heaviest fruit (365.33 g) was recorded in MI16. Lodh et al. (1974) and lqbal et al. (1995) also reported the variation of fruit weight among the different mango varieties. This variation may be due to genetic or physiological factors. A wide range of variation was observed among the germplasm in respect of fruit length. MI16 produced the longest fruit $(11.50 \mathrm{~cm})$ followed by MI24 $(10.74 \mathrm{~cm})$ and MI27 $(10.49 \mathrm{~cm})$. The average breadth of different mango germplasm was found to vary from 10.96 to $5.37 \mathrm{~cm}$ (Table $4 \mathrm{~b})$. The thickest fruit $(9.71 \mathrm{~cm})$ was in Ml16, whereas MI60 occupied lowest thickness $(4.29 \mathrm{~cm})$.

\section{Table 4a. Fruit characteristics of 60 mango genotypes}

\begin{tabular}{|c|c|c|c|c|c|}
\hline Genotypes & Fruit shape & Skin texture & $\begin{array}{l}\text { Days to maturity } \\
\text { (days) }\end{array}$ & $\begin{array}{c}\text { Fruit } \\
\text { harvest/panicle }\end{array}$ & $\begin{array}{l}\text { No. of harvested } \\
\text { fruit/plant }\end{array}$ \\
\hline MI01 & Elliptic & Smooth & 172.00 & 4.50 & 54.33 \\
\hline $\mathrm{MIO} 2$ & Oblong & Intermediate & 171.33 & 4.32 & 53.33 \\
\hline $\mathrm{MIO3}$ & Elliptic & Intermediate & 135.67 & 3.22 & 46.67 \\
\hline $\mathrm{MIO4}$ & Elliptic & Smooth & 162.67 & 4.88 & 58.33 \\
\hline MI08 & Oblong & Smooth & 140.00 & 2.02 & 41.33 \\
\hline MI09 & Oblong & Smooth & 129.00 & 4.25 & 53.33 \\
\hline MI12 & Oblong & Smooth & 117.00 & 4.55 & 56.33 \\
\hline MI16 & Oblong & Rough & 141.67 & 1.62 & 24.00 \\
\hline MI19 & Round & Rough & 135.00 & 2.37 & 32.00 \\
\hline MI20 & Elliptic & Smooth & 161.67 & 2.23 & 51.33 \\
\hline MI21 & Oblong & Smooth & 154.67 & 2.10 & 43.67 \\
\hline MI22 & Elliptic & Intermediate & 136.67 & 1.68 & 24.00 \\
\hline MI23 & Oblong & Smooth & 121.33 & 3.90 & 42.33 \\
\hline MI24 & Elliptic & Smooth & 121.67 & 4.76 & 54.00 \\
\hline MI25 & Oblong & Smooth & 173.33 & 4.28 & 54.33 \\
\hline MI26 & Roundish & Smooth & 134.00 & 4.25 & 34.00 \\
\hline MI27 & Elliptic & Smooth & 127.67 & 2.05 & 51.00 \\
\hline MI28 & Elliptic & Smooth & 178.67 & 5.23 & 60.33 \\
\hline MI29 & Elliptic & Smooth & 137.67 & 1.59 & 23.33 \\
\hline MI33 & Oblong & Smooth & 130.33 & 1.95 & 27.00 \\
\hline MI38 & Elliptic & Smooth & 125.00 & 3.37 & 53.33 \\
\hline MI39 & Oblong & Rough & 129.67 & 1.92 & 36.00 \\
\hline $\mathrm{MI} 40$ & Round & Smooth & 145.33 & 3.41 & 44.00 \\
\hline MI41 & Elliptic & Intermediate & 131.00 & 1.56 & 23.33 \\
\hline MI43 & Elliptic & Intermediate & 175.00 & 2.09 & 25.33 \\
\hline Ml44 & Oblong & Rough & 137.00 & 4.84 & 39.67 \\
\hline MI45 & Oblong & Smooth & 128.00 & 4.22 & 50.00 \\
\hline MI46 & Elliptic & Smooth & 160.67 & 4.22 & 52.00 \\
\hline MI47 & Round & Smooth & 132.00 & 2.72 & 33.00 \\
\hline MI48 & Oblong & Rough & 136.00 & 2.45 & 23.67 \\
\hline MI49 & Elliptic & Smooth & 171.33 & 2.03 & 39.00 \\
\hline MI50 & Round & Smooth & 140.33 & 1.06 & 23.00 \\
\hline
\end{tabular}


Table 4a. Contd.

\begin{tabular}{|l|l|l|c|c|c|}
\hline Genotypes & Fruit shape & Skin texture & $\begin{array}{c}\text { Days to maturity } \\
\text { (days) }\end{array}$ & $\begin{array}{c}\text { Fruit } \\
\text { harvest/panicle }\end{array}$ & $\begin{array}{c}\text { No. of harvested } \\
\text { fruit/plant }\end{array}$ \\
\hline MI51 & Round & Smooth & 146.33 & 3.48 & 23.67 \\
\hline MI52 & Oblong & Smooth & 157.67 & 3.83 & 36.67 \\
\hline MI54 & Elliptic & Smooth & 131.00 & 2.20 & 37.33 \\
\hline MI58 & Roundish & Smooth & 127.00 & 1.58 & 21.33 \\
\hline MI60 & Oblong & Smooth & 125.67 & 1.78 & 23.67 \\
\hline MI61 & Elliptic & Smooth & 138.67 & 3.37 & 49.00 \\
\hline MI64 & Oblong & Smooth & 119.33 & 4.39 & 53.00 \\
\hline MI70 & Round & Intermediate & 142.33 & 3.27 & 31.00 \\
\hline MI74 & Elliptic & Smooth & 121.67 & 3.22 & 52.00 \\
\hline MI75 & Oblong & Smooth & 169.00 & 2.30 & 23.00 \\
\hline MI77 & Elliptic & Smooth & 123.00 & 3.29 & 23.00 \\
\hline MI80 & Elliptic & Intermediate & 150.33 & 2.69 & 38.67 \\
\hline MI81 & Oblong & Smooth & 168.00 & 2.60 & 36.67 \\
\hline MI82 & Elliptic & Smooth & 123.67 & 2.83 & 26.00 \\
\hline MI83 & Oblong & Smooth & 128.00 & 2.35 & 24.00 \\
\hline MI84 & Roundish & Smooth & 131.00 & 2.35 & 24.00 \\
\hline MI85 & Elliptic & Smooth & 148.00 & 3.29 & 37.00 \\
\hline MI86 & Elliptic & Intermediate & 162.00 & 2.04 & 26.33 \\
\hline MI88 & Oblong & Smooth & 127.00 & 3.23 & 35.00 \\
\hline MI90 & Roundish & Rough & 169.67 & 2.10 & 35.00 \\
\hline MI91 & Oblong & Smooth & 156.33 & 3.12 & 54.67 \\
\hline MI92 & Oblong & Rough & 140.33 & 3.48 & 44.33 \\
\hline MI93 & Round & Smooth & 123.33 & 1.86 & 24.67 \\
\hline MI94 & Elliptic & Smooth & 120.00 & 5.46 & 59.33 \\
\hline MI95 & Elliptic & Smooth & 148.00 & 4.31 & 54.33 \\
\hline MI96 & Oblong & Smooth & 120.33 & 3.28 & 28.67 \\
\hline M197 & Elliptic & Smooth & 120.00 & 1.80 & 22.33 \\
\hline MI98 & Roundish & Smooth & 122.67 & 2.33 & 48.00 \\
\hline Range & - & - & $117.00-78.67$ & $1.56-5.46$ & $21.33-60.33$ \\
\hline Mean & - & - & 7.09 & 13.07 & 38.68 \\
\hline CV (\%) & - & - & 5.78 & 0.55 & 11.29 \\
\hline SE & - & - & 17.71 & 0.88 & 2.52 \\
\hline LSD(0.01) & - & - & & 9.33 \\
\hline & & & & \\
\hline
\end{tabular}

Table $\mathbf{4 b}$. Fruit characteristics of $\mathbf{6 0}$ mango genotypes

\begin{tabular}{|l|c|c|c|c|c|l|l|l|}
\hline Genotypes & $\begin{array}{c}\text { Fruit weight } \\
(\mathrm{g})\end{array}$ & $\begin{array}{c}\text { Fruit length } \\
(\mathrm{cm})\end{array}$ & $\begin{array}{c}\text { Fruit } \\
\text { breadth } \\
(\mathrm{cm})\end{array}$ & $\begin{array}{c}\text { Fruit } \\
\text { thickness } \\
(\mathrm{cm})\end{array}$ & $\begin{array}{c}\text { Wt. of } \\
\text { harvested } \\
\text { fruit/ Plant }\end{array}$ & $\begin{array}{c}\text { Pulp } \\
\text { texture }\end{array}$ & Pulp colour & Fibrousness \\
\hline MI01 & 245.00 & 8.65 & 5.37 & 5.50 & 13.11 & Firm & Light orange & Scarce \\
\hline MI02 & 327.33 & 9.25 & 8.42 & 7.17 & 7.68 & Soft & Light orange & Abundant \\
\hline MI03 & 327.00 & 9.41 & 7.21 & 6.02 & 11.51 & Firm & Yellow & Abundant \\
\hline MI04 & 317.00 & 9.66 & 9.44 & 7.81 & 17.99 & Firm & Yellow & Scarce \\
\hline MI08 & 236.33 & 7.78 & 7.40 & 5.31 & 15.56 & Juicy & Yellow & Scarce \\
\hline MI09 & 338.00 & 10.11 & 7.32 & 7.25 & 11.29 & Firm & Yellow & Scarce \\
\hline MI12 & 245.67 & 8.65 & 6.45 & 5.74 & 18.35 & Juicy & Yellow & Scarce \\
\hline MI16 & 365.33 & 11.50 & 10.96 & 9.71 & 8.02 & Firm & Yellow & Abundant \\
\hline MI19 & 323.33 & 8.69 & 9.40 & 5.63 & 8.83 & Firm & Yellow & Abundant \\
\hline MI20 & 244.00 & 8.93 & 6.29 & 5.71 & 11.02 & Soft & Yellow & Scarce \\
\hline MI21 & 219.00 & 8.59 & 6.33 & 6.70 & 9.67 & Soft & Light yellow & Scarce \\
\hline MI22 & 242.00 & 7.94 & 9.48 & 7.11 & 7.64 & Firm & Yellow & Abundant \\
\hline MI23 & 245.33 & 7.86 & 6.23 & 5.27 & 8.08 & Soft & Bright yellow & Scarce \\
\hline MI24 & 353.00 & 10.74 & 7.88 & 8.23 & 18.68 & Soft & Yellow & Scarce \\
\hline MI25 & 317.33 & 9.97 & 7.36 & 6.79 & 10.74 & Soft & Yellow & Abundant \\
\hline
\end{tabular}


Table 4b. Contd.

\begin{tabular}{|c|c|c|c|c|c|c|c|c|}
\hline Genotypes & $\begin{array}{l}\text { Fruit weight } \\
\text { (g) }\end{array}$ & $\begin{array}{l}\text { Fruit } \\
\text { length } \\
(\mathrm{cm})\end{array}$ & $\begin{array}{l}\text { Fruit } \\
\text { breadth } \\
\text { (cm) }\end{array}$ & $\begin{array}{c}\text { Fruit } \\
\text { thickness } \\
(\mathrm{cm})\end{array}$ & $\begin{array}{c}\text { Wt. of } \\
\text { harvested } \\
\text { fruit/Plant }\end{array}$ & $\begin{array}{l}\text { Pulp } \\
\text { texture }\end{array}$ & Pulp colour & Fibrousness \\
\hline MI26 & 324.00 & 8.75 & 6.40 & 5.78 & 8.39 & Soft & Yellow & Scarce \\
\hline MI27 & 319.00 & 10.49 & 8.51 & 6.19 & 7.91 & Soft & Yellow & Scarce \\
\hline MI28 & 249.33 & 8.27 & 6.46 & 5.85 & 23.12 & Soft & Bright yellow & Scarce \\
\hline MI29 & 233.00 & 8.14 & 6.37 & 5.84 & 7.83 & Soft & Yellow & Scarce \\
\hline MI33 & 234.33 & 8.34 & 7.07 & 6.35 & 8.57 & Juicy & Yellow & Abundant \\
\hline MI38 & 253.67 & 8.53 & 7.56 & 6.64 & 7.94 & Juicy & Orange & Scarce \\
\hline MI39 & 264.67 & 8.73 & 7.91 & 7.04 & 10.19 & Firm & Orange & Much \\
\hline MI40 & 287.00 & 7.79 & 6.17 & 5.81 & 14.88 & Juicy & Yellow & Abundant \\
\hline MI41 & 242.00 & 8.73 & 6.76 & 6.63 & 7.27 & Soft & Yellow & \begin{tabular}{|l|} 
Abundant \\
\end{tabular} \\
\hline MI43 & 332.33 & 9.39 & 6.28 & 5.49 & 9.06 & Juicy & Yellow & Much \\
\hline MI44 & 257.33 & 8.46 & 7.33 & 5.18 & 10.36 & Firm & Orange & Scarce \\
\hline MI45 & 256.67 & 8.80 & 7.60 & 6.51 & 13.64 & Firm & Yellow & Scarce \\
\hline MI46 & 355.00 & 10.12 & 9.71 & 5.63 & 23.47 & Firm & Orange & Scarce \\
\hline MI47 & 255.67 & 9.99 & 6.52 & 5.63 & 7.44 & Soft & Yellow & Scarce \\
\hline MI48 & 243.00 & 9.15 & 5.61 & 5.90 & 7.48 & Soft & Yellow & Scarce \\
\hline MI49 & 217.67 & 7.45 & 6.46 & 6.82 & 7.36 & Soft & Yellow & Scarce \\
\hline MI50 & 313.00 & 9.63 & 6.59 & 7.38 & 7.11 & Soft & Yellow & Abundant \\
\hline MI51 & 314.67 & 9.01 & 7.35 & 6.47 & 7.67 & Juicy & Yellow & Much \\
\hline MI52 & 355.00 & 8.63 & 7.17 & 5.83 & 11.73 & Soft & Orange & Scare \\
\hline MI54 & 321.67 & 10.33 & 5.55 & 5.10 & 12.01 & Soft & Yellow & Scare \\
\hline MI58 & 256.33 & 9.12 & 5.63 & 5.51 & 8.17 & Soft & Yellow & Scare \\
\hline MI60 & 332.33 & 8.67 & 5.90 & 4.29 & 8.30 & Soft & Yellow & Scare \\
\hline MI61 & 320.00 & 8.25 & 6.38 & 5.19 & 10.72 & Soft & Orange & Scare \\
\hline MI64 & 248.67 & 8.65 & 7.74 & 8.22 & 10.92 & Juicy & Yellow & Abundant \\
\hline MI70 & 332.00 & 10.07 & 9.19 & 8.28 & 10.96 & Firm & Yellow & Scare \\
\hline MI74 & 247.33 & 9.08 & 7.72 & 5.49 & 17.64 & Juicy & Orange & \begin{tabular}{|l|} 
Abundant \\
\end{tabular} \\
\hline MI75 & 320.67 & 7.77 & 6.89 & 5.88 & 8.49 & Soft & Cream & Scare \\
\hline MI77 & 320.33 & 8.32 & 6.50 & 6.86 & 9.51 & Firm & Orange & Much \\
\hline MI80 & 273.00 & 8.15 & 5.87 & 6.29 & 6.33 & Firm & Orange & Abundant \\
\hline MI81 & 325.00 & 8.46 & 7.88 & 5.66 & 9.42 & Soft & Yellow & Abundant \\
\hline Ml82 & 266.00 & 8.64 & 6.10 & 5.26 & 7.30 & Juicy & Pale yellow & Abundant \\
\hline MI83 & 239.00 & 8.32 & 6.80 & 6.56 & 6.76 & Firm & Yellow & Scare \\
\hline MI84 & 229.00 & 6.86 & 5.80 & 4.97 & 6.93 & Soft & Orange & Scare \\
\hline MI85 & 263.67 & 8.37 & 6.63 & 5.85 & 11.46 & Firm & Pinkish yellow & Scare \\
\hline MI86 & 254.00 & 8.15 & 5.48 & 5.30 & 7.21 & Soft & Yellow & Scare \\
\hline MI88 & 257.67 & 8.20 & 7.39 & 7.15 & 8.99 & Firm & Yellow & Scare \\
\hline MI90 & 236.33 & 8.28 & 6.08 & 5.60 & 11.47 & Soft & Yellow & Much \\
\hline MI91 & 233.33 & 8.53 & 8.53 & 6.74 & 8.37 & Soft & Orange & Much \\
\hline MI92 & 273.67 & 9.54 & 6.37 & 5.80 & 12.14 & Firm & Orange & Much \\
\hline MI93 & 231.67 & 9.48 & 5.82 & 5.19 & 6.47 & Juicy & Yellow & Scare \\
\hline MI94 & 313.33 & 10.47 & 9.36 & 8.51 & 25.04 & Firm & Yellow & Much \\
\hline MI95 & 355.33 & 9.63 & 8.55 & 8.69 & 16.30 & Firm & Yellow & Much \\
\hline MI96 & 233.00 & 8.46 & 7.34 & 7.70 & 7.64 & Firm & Orange & Abundant \\
\hline MI97 & 273.67 & 10.28 & 10.09 & 6.95 & 7.18 & Soft & Bright yellow & Scare \\
\hline M198 & 337.33 & 8.20 & 6.24 & 7.14 & 7.41 & Soft & Yellow & Scare \\
\hline Range & $\begin{array}{l}365.33- \\
219.00\end{array}$ & $11.50-6.86$ & $\begin{array}{c}10.96- \\
5.37\end{array}$ & $9.71-5.10$ & $25.04-6.33$ & - & - & - \\
\hline Mean & 283.36 & 8.92 & 7.19 & 6.36 & 10.71 & - & - & - \\
\hline CV (\%) & 6.12 & 8.05 & 5.16 & 7.26 & 9.86 & - & - & - \\
\hline SE & 10.01 & 0.42 & 0.21 & 0.27 & 0.61 & - & - & - \\
\hline $\mathrm{LSD}(0.01)$ & 37.08 & 1.54 & 0.79 & 0.99 & 2.26 & - & - & - \\
\hline
\end{tabular}




\section{Pulp characteristics}

Among the 60 genotypes 28 showed soft pulp texture and 21 genotypes showed firm and rest genotype were juicy type pulp textures (Table 5). Most of the genotypes had yellow pulp color. Very few genotypes showed abundant or much fibrous, while most of them were least fibrous. Per cent edible portion of fruit is an important character for selecting quality fruit. The present study, edible portion significantly varied from 45.22 to $79.83 \%$ (Table 5). The highest percentage of non-edible portion (54.78\%) was obtained from MI39, whereas the germplasm MI09 had the lowest percentage of non-edible portion (20.17\%). The present investigation is in partial agreement with the research findings of Kabir et al. (2007) who recorded 20.95 to $55.06 \%$ non-edible portion from 12 mango germplasm. Total soluble solid contents were measured at the eating ripe stage, and it was observed that the variation in TSS among the germplasm was highly significant. MI28 contained the highest TSS (28.26\% Brix) and MI20 contained the lowest TSS $(16.90 \%$ Brix)). The present findings agreed with the results of Kabir et al. (2007) who found 16.25 to $27.65 \%$ Brix while studying 12 mango germplasm under Mymensingh conditions.

Table 5. Pulp and stone characteristics of 60 mango genotypes

\begin{tabular}{|c|c|c|c|c|c|c|c|c|}
\hline Genotypes & $\begin{array}{c}\text { Edible } \\
\text { portion } \\
(\%)\end{array}$ & $\begin{array}{c}\text { Non-edible } \\
\text { portion } \\
(\%)\end{array}$ & $\begin{array}{c}\text { Total } \\
\text { soluble } \\
\text { solid (\%) }\end{array}$ & $\begin{array}{c}\text { Weight of } \\
\text { stone } \\
\text { (gm) }\end{array}$ & $\begin{array}{l}\text { Length of } \\
\text { Stone } \\
(\mathrm{cm})\end{array}$ & $\begin{array}{l}\text { Breadth of } \\
\text { the stone } \\
(\mathrm{cm})\end{array}$ & $\begin{array}{l}\text { Thickness of } \\
\text { stone } \\
(\mathrm{cm})\end{array}$ & $\begin{array}{l}\text { Veins of the } \\
\text { stone }\end{array}$ \\
\hline MI01 & 69.31 & 30.69 & 27.50 & 23.65 & 9.63 & 3.01 & 1.42 & Depressed \\
\hline MI02 & 72.17 & 27.83 & 23.33 & 45.71 & 7.43 & 4.29 & 1.75 & Elevated \\
\hline MI03 & 68.46 & 31.54 & 26.24 & 37.20 & 5.73 & 3.60 & 2.30 & Elevated \\
\hline MI04 & 76.92 & 23.08 & 20.08 & 50.62 & 6.66 & 4.18 & 1.85 & Depressed \\
\hline MI08 & 73.66 & 26.34 & 24.09 & 50.03 & 9.30 & 4.60 & 2.26 & Level \\
\hline MI09 & 79.83 & 20.17 & 21.08 & 51.92 & 9.59 & 5.13 & 2.37 & Depressed \\
\hline MI12 & 69.21 & 30.79 & 24.87 & 53.62 & 7.27 & 4.76 & 2.34 & Level \\
\hline MI16 & 70.78 & 29.22 & 24.77 & 47.01 & 12.93 & 5.84 & 1.95 & Elevated \\
\hline MI19 & 73.91 & 26.09 & 18.75 & 44.07 & 6.94 & 3.95 & 1.85 & Depressed \\
\hline MI20 & 79.52 & 20.48 & 16.90 & 38.44 & 7.59 & 3.20 & 1.62 & Elevated \\
\hline MI21 & 61.58 & 38.42 & 23.33 & 36.12 & 6.68 & 3.78 & 1.96 & \begin{tabular}{|l|} 
Elevated \\
\end{tabular} \\
\hline MI22 & 71.73 & 28.27 & 21.62 & 47.06 & 7.33 & 5.82 & 2.09 & Elevated \\
\hline MI23 & 59.40 & 40.60 & 21.97 & 40.09 & 7.73 & 3.38 & 2.22 & Depressed \\
\hline \begin{tabular}{|l|} 
MI24 \\
\end{tabular} & 78.00 & 22.00 & 21.32 & 36.55 & 11.62 & 3.70 & 2.64 & Elevated \\
\hline MI25 & 62.98 & 37.02 & 23.42 & 41.96 & 7.71 & 4.02 & 2.03 & Depressed \\
\hline MI26 & 69.56 & 30.44 & 22.35 & 33.09 & 7.53 & 3.93 & 1.75 & Elevated \\
\hline MI27 & 78.00 & 22.00 & 16.91 & 27.67 & 10.88 & 3.30 & 1.71 & \begin{tabular}{|l|} 
Elevated \\
\end{tabular} \\
\hline MI28 & 76.07 & 23.93 & 28.26 & 27.08 & 5.50 & 3.28 & 1.64 & Elevated \\
\hline \begin{tabular}{|l|} 
MI29 \\
\end{tabular} & 57.96 & 42.04 & 21.93 & 28.68 & 5.81 & 3.81 & 2.27 & Elevated \\
\hline \begin{tabular}{|l|} 
MI33 \\
\end{tabular} & 52.40 & 47.60 & 22.15 & 77.08 & 6.09 & 3.38 & 1.71 & Depressed \\
\hline MI38 & 65.24 & 34.76 & 22.88 & 36.62 & 5.88 & 3.60 & 2.30 & Elevated \\
\hline MI39 & 45.22 & 54.78 & 24.88 & 111.61 & 11.54 & 4.66 & 2.86 & Elevated \\
\hline MI40 & 52.71 & 47.29 & 22.34 & 75.55 & 6.02 & 4.65 & 2.32 & Elevated \\
\hline MI41 & 66.64 & 33.36 & 22.92 & 25.50 & 5.72 & 3.84 & 1.61 & Depressed \\
\hline MI43 & 66.05 & 33.95 & 23.20 & 24.06 & 6.24 & 3.33 & 1.69 & Depressed \\
\hline MI44 & 57.98 & 42.02 & 25.20 & 43.57 & 7.24 & 3.74 & 2.23 & Elevated \\
\hline Ml45 & 54.74 & 45.26 & 22.94 & 37.60 & 5.75 & 3.92 & 2.27 & Elevated \\
\hline MI46 & 67.63 & 32.37 & 22.14 & 34.29 & 12.24 & 4.61 & 2.25 & Depressed \\
\hline MI47 & 70.17 & 29.83 & 21.07 & 72.97 & 8.94 & 5.48 & 2.27 & Elevated \\
\hline MI48 & 55.72 & 44.28 & 24.68 & 34.25 & 6.23 & 3.96 & 1.59 & Elevated \\
\hline MI49 & 66.28 & 33.72 & 22.68 & 40.63 & 7.64 & 3.71 & 1.96 & Elevated \\
\hline MI50 & 64.50 & 35.50 & 22.11 & 43.16 & 5.41 & 5.18 & 1.99 & Elevated \\
\hline MI51 & 54.51 & 45.49 & 22.55 & 51.68 & 6.54 & 5.79 & 2.54 & Elevated \\
\hline MI52 & 56.81 & 43.19 & 22.10 & 44.84 & 7.83 & 3.86 & 1.87 & \begin{tabular}{|l|} 
Elevated \\
\end{tabular} \\
\hline MI54 & 73.85 & 26.15 & 20.25 & 21.33 & 7.82 & 4.10 & 1.72 & \begin{tabular}{|l|} 
Elevated \\
\end{tabular} \\
\hline MI58 & 55.66 & 44.34 & 21.35 & 31.44 & 5.34 & 3.45 & 1.76 & Level \\
\hline MI60 & 48.64 & 51.36 & 22.16 & 49.85 & 8.89 & 3.66 & 2.38 & Depressed \\
\hline
\end{tabular}


Table 5. Contd.

\begin{tabular}{|c|c|c|c|c|c|c|c|c|}
\hline Genotypes & $\begin{array}{c}\text { Edible } \\
\text { portion } \\
(\%)\end{array}$ & $\begin{array}{l}\text { Non-edible } \\
\text { portion } \\
(\%)\end{array}$ & $\begin{array}{c}\text { Total } \\
\text { soluble } \\
\text { solid (\%) }\end{array}$ & $\begin{array}{l}\text { Weight of } \\
\text { stone } \\
\text { (gm) }\end{array}$ & $\begin{array}{l}\text { Length of } \\
\text { Stone } \\
(\mathrm{cm})\end{array}$ & $\begin{array}{l}\text { Breadth of } \\
\text { the stone } \\
(\mathrm{cm})\end{array}$ & $\begin{array}{l}\text { Thickness } \\
\text { of stone } \\
\text { (cm) }\end{array}$ & $\begin{array}{l}\text { Veins of the } \\
\text { stone }\end{array}$ \\
\hline MI61 & 58.06 & 41.94 & 27.54 & 39.51 & 6.75 & 3.79 & 1.75 & Elevated \\
\hline MI64 & 65.87 & 34.13 & 23.56 & 35.79 & 6.86 & 3.51 & 2.39 & Elevated \\
\hline MI70 & 59.97 & 40.03 & 23.68 & 65.54 & 9.25 & 5.72 & 2.27 & Elevated \\
\hline MI74 & 66.65 & 33.35 & 24.70 & 45.43 & 7.71 & 3.80 & 2.20 & Elevated \\
\hline MI75 & 55.69 & 44.31 & 26.18 & 26.47 & 7.78 & 3.85 & 1.66 & Elevated \\
\hline MI77 & 62.08 & 37.92 & 24.70 & 35.74 & 9.09 & 4.86 & 2.46 & Level \\
\hline Ml80 & 65.71 & 34.29 & 25.40 & 23.64 & 5.64 & 3.48 & 1.80 & Depressed \\
\hline MI81 & 53.44 & 46.56 & 23.09 & 44.05 & 9.45 & 4.76 & 2.73 & Depressed \\
\hline MI82 & 46.44 & 53.56 & 26.96 & 96.11 & 6.77 & 3.80 & 1.79 & Elevated \\
\hline Ml83 & 61.43 & 38.57 & 25.83 & 38.25 & 6.82 & 3.68 & 2.06 & Elevated \\
\hline MI84 & 53.68 & 46.32 & 26.05 & 52.47 & 6.69 & 4.48 & 1.80 & Elevated \\
\hline MI85 & 52.04 & 47.96 & 25.39 & 87.04 & 5.44 & 3.48 & 1.70 & Elevated \\
\hline MI86 & 58.85 & 41.15 & 24.33 & 65.30 & 12.53 & 5.51 & 1.60 & Elevated \\
\hline MI88 & 65.72 & 34.28 & 22.70 & 30.65 & 8.30 & 4.59 & 1.76 & Level \\
\hline MI90 & 57.33 & 42.67 & 25.49 & 31.60 & 5.87 & 4.01 & 2.41 & Elevated \\
\hline MI91 & 69.93 & 30.07 & 24.11 & 61.37 & 8.66 & 4.35 & 2.35 & Elevated \\
\hline \begin{tabular}{|l|}
$\mathrm{Ml92}$ \\
\end{tabular} & 52.76 & 47.24 & 24.21 & 73.43 & 11.13 & 4.71 & 2.55 & Elevated \\
\hline MI93 & 56.05 & 43.95 & 23.64 & 40.16 & 5.58 & 3.90 & 1.98 & Elevated \\
\hline MI94 & 77.26 & 22.74 & 19.22 & 30.88 & 9.83 & 4.88 & 2.55 & Elevated \\
\hline \begin{tabular}{|l|} 
MI95 \\
\end{tabular} & 74.03 & 25.97 & 17.56 & 38.17 & 9.92 & 4.85 & 2.37 & Elevated \\
\hline MI96 & 68.70 & 31.30 & 18.26 & 50.22 & 9.52 & 4.61 & 2.20 & Elevated \\
\hline \begin{tabular}{|l|}
$\mathrm{Ml} 97$ \\
\end{tabular} & 66.09 & 33.91 & 23.33 & 60.04 & 10.62 & 3.78 & 1.99 & Elevated \\
\hline \begin{tabular}{|l|} 
MI98 \\
\end{tabular} & 48.36 & 51.64 & 21.96 & 92.07 & 8.03 & 4.72 & 1.84 & Depressed \\
\hline Range & $45.22-79.83$ & $20.17-54.78$ & $16.90-28.26$ & $21.33-111.61$ & $5.34-12.93$ & $3.01-5.84$ & $1.42-2.86$ & - \\
\hline Mean & $\begin{array}{l}63.67 \\
\end{array}$ & 36.33 & 23.07 & \begin{tabular}{|l|}
46.02 \\
\end{tabular} & 7.80 & 4.20 & 2.06 & - \\
\hline CV (\%) & 2.9 & 5.09 & 4.85 & 4.35 & 5.88 & 9.17 & 8.62 & - \\
\hline SE & 1.07 & 1.07 & 0.65 & 1.16 & 0.27 & 0.22 & 0.10 & - \\
\hline $\operatorname{LSD}(0.01)$ & 3.96 & 3.96 & 2.39 & 4.28 & 0.98 & 0.82 & 0.38 & - \\
\hline
\end{tabular}

\section{Stone characteristics}

The results showed that MI39 produced the heaviest stone $(111.61 \mathrm{~g})$ followed by MI82 $(96.11 \mathrm{~g})$ and MI98 (92.07 g) (Table 5). The lightest was observed in MI54 $(21.33 \mathrm{~g})$. The results of the present study are in partial agreement with the research findings of Haque et al (1993) who recorded 21.14 to $98.53 \mathrm{~g}$ stone weight. Average number of stone length of different germplasm exhibited wide variations. MI16 had the longest stone $(12.93 \mathrm{~cm}$.) but MI58 produced the smallest stone $(5.34 \mathrm{~cm})$. The widest stone $(5.84$ $\mathrm{cm}$ ) was noted in MI16 and the thinnest stone $(1.42 \mathrm{~cm}$ ) was found in MI01 (Table 5). Saha et al. (1998) observed similar variation on the stone sizes of mango. Most of the genotypes showed elevated veins on stone; only five genotypes showed level type veins and rest were depressed type veined stone. Among the genotypes only fourteen had no fibre on the stone and maximum genotypes showed fibrousness in stone. Considering all of the characteristics under study, some promising genotypes viz. MI94, MI46, MI28 and MI24 were selected for further evaluation with a view to develop new variety(s) of mango.

\section{Conclusion}

The development of mango variety through the selection is important. The study on physio-morphological, floral biological and fruit characteristics of mango showed that there have variations among the germplasm. This gives the opportunity to select germplasm on the basis of desirable characters. The improved variety of mango may be developed using the variability of collected genotypes. 


\section{Acknowledgments}

This research work was financially supported by the Prime-Minister's Advanced Studies and Research Scholarships from the Prime-Minister Office, Government of the People's Republic of Bangladesh.

\section{References}

De Candolle, A. 1904. Origin of Cultivated Plants. Kegan Paul, Trench, London.

Haque, A.M.M.M., Ali, M.R., Uddin, M.R. and Hossain, A.K.M.A. 1993. Evaluation of elite mango cultivars at southern region of Bangladesh. Bangladesh J. Plant Breed. Genet., 6(2): 21-28.

Hodgson, R.W. 1967. Horticultural varieties of citrus. The Citrus Industry (W. Reuther, H. J. Webber, and L. D. Batchelor. eds.). University of California Press, Berkely, 1: 431-591.

Hossain, A.K.M.A. 1989. Manual on Mango Cultivation in Bangladesh. Horticulture Division, Bangladesh Agricultural Research Institute, Joydebpur, Gazipur, Bangladesh. pp. 39-58.

IPGRI. 2003. Catalogue on mango germplasm. Horticulture Research Centre. Bangladesh Agricultural Research Institute, Joydebpur, Gazipur-1701, Bangladesh. 1: 2-7.

Iqbal, S.M., Uddin, M.S. and Shaku, M.A. 1995. Performance of exotic mango germplasm under Bangladesh conditions. In: Annual Report on Mango Improvement (1994-95), Regional Horticultural Research Station, Bangladesh Agricultural Research Institute, Nawabgonj. pp. 1-9.

Islam, M.S., Bhuyan, M.A.J., Biswas, M., Islam, M.N. and Hossain, M.A. 1995. Studies on the growth, flowering and fruit characteristics of eight mango cultivars. Bangladesh Hort., 23(1\&2): 59-65.

Kabir, M.A., Rahim, M.A. and Majumder, D.A.N. 2007. Studies on the Physico-Chemical characteristics of some mango germplasm. J. Agrof. Evn., 1(1): 147-152.

Lodh, S.B., Subramanyam, M.D. and Divakar, N.G. 1974. Physico-chemical studies of some important mango verities. Indian J. Hort., 31(2): 160-161.

Majumder,P.K. and Sharma, D.K. 1990. Mango. In: Fruits: Tropical and Sub-tropical ( T.K. Bose and S.K. Mitra Ed.), Nayaprokash, Calcutta, India.pp.1-62.

Mukherjee, S.K. 1997. Introduction: Botany and importance. In: The mango Botany, Production and Uses $1^{\text {st }}$ edition (R. E. Litz Ed.), CAB International, Wallingford, UK. pp. 1-19.

Popenoe, W. 1927. Manual of tropical and sub-tropical fruits, Macmillan, New York, USA.

Saha, N.N., Bhuyan, M.A.J. and Islam, M.S. 1995. Variability in early and mid seasons mango varieties grown in Bangladesh. Annals Bangladesh Agric., 5(2): 135-138.

Sardar, P.K., Hossain M.A., Islam M.S. and Khondaker, S.M.A.T. 1998. Studies on the physico-morphological characters of some popular mango cultivars. Bangladesh J. Agril. Sci., 25 (1): 1-4. 